
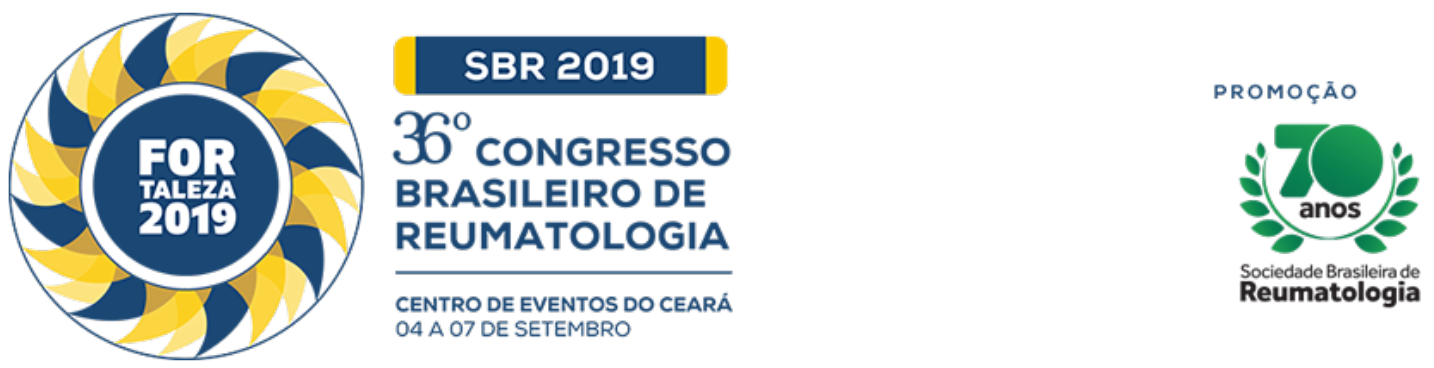

\title{
HEPATIC FAILURE AT THE DIAGNOSIS OF JUVENILE SYSTEMIC LUPUS ERYTHEMATOSUS
}

Aline Garcia Islabão (Hospital da Criança de Brasília, BRASILIA, DF, Brasil), Sarah Polyane Silva Veloso (Hospital da Criança de Brasília, BRASILIA, DF, Brasil), Regiane Duque Minardi Neves (Hospital da Criança de Brasília, BRASILIA, DF, Brasil), Maria Custodia Machado Ribeiro (Hospital da Criança de Brasília, BRASILIA, DF, Brasil), Marlon Sousa Lopes (Hospital da Criança de Brasília, BRASILIA, DF, Brasil), Maria Inês Minardi Neves (UniCEUB, BRASILIA, DF, Brasil)

\section{BACKGROUND}

Systemic lupus erythematosus (SLE) is a chronic disease that generates an inflammatory state of the connective tissue, affecting several organs and systems. Liver involvement may be due to lupus hepatitis, autoimmune hepatitis, primary biliary cirrhosis, cholangitis, viral hepatitis, and adverse effects of medications used to treat SLE. Lupus hepatitis should be considered only after excluding other etiological factors. The clinical presentation is usually asymptomatic or sub-clinical, and may mimic, in some cases, acute viral hepatitis. Although extraordinary, liver involvement generates high rates of infant mortality.

\section{CASE REPORT}

Adolescent, 11 years old, female, has been with jaundice, vomiting, asthenia, fever, anorexia and polyarticular arthritis, symmetrical and additive for 4 months. Initiated investigation of icteric syndrome, with initial suspicion of hepatitis. She presented somnolence compatible with hepatic encephalopathy. Laboratory results: autoimmune hemolytic anemia, thrombocytopenia, elevated transaminases, gamma glutamyl transferase and alkaline phosphatase, hyperbilirubinemia, hypocomplementemia, hypoalbuminemia, extended TAP, INR of 1.8 and hypergammaglobulinemia; serologies for negative viral hepatitis; Anti-LKM, Anti-TTG, anti-smooth muscle non-reactants; Anti-DNA positive and FAN> 1/640 Homogeneous Nuclear. Abdominal ultrasonography showed non-specific periportal diffuse hyperechogenicity associated with mild ascites and mild intra-hepatic biliary dilatation and splenomegaly. She was diagnosed with Systemic Lupus Erythematosus (SLEDAI 10) and acute liver failure.

\section{CONCLUSION}

The case presented shows the diagnosis of lupus from acute liver failure. This condition is extremely rare in lupus patients, and is even more difficult to perform as the initial manifestation of the disease. Hepatic impairment in SLE may be related to the positivity of the ribosomal anti-P antibody, which was not present in the patient. The presence of anti-DNA assists in the differential diagnosis between autoimmune hepatitis and SLE, as it is present in patients with lupus. Usually, liver dysfunction in SLE is mild and has a good response to the use of steroids and immunosuppressants such as azathioprine. 